\title{
Evaluating the Effect of Spiking Network Parameters on Polychronization
}

\author{
Panagiotis Ioannou, Matthew Casey and André Grüning \\ Department of Computing, University of Surrey, Guildford, Surrey, GU2 7XH, UK
}

\begin{abstract}
Spiking neural networks (SNNs) are considered to be more biologically realistic compared to typical rate-coded networks as they can model closely different types of neurons and their temporal dynamics. Typical spiking models use a number of fixed parameters such as the ratio between excitatory and inhibitory neurons. However, the parameters that are used in these models focus almost exclusively on our understanding of the neocortex with, for example, $80 \%$ of neurons chosen as excitatory and $20 \%$ inhibitory. In this paper we will evaluate how varying the ratio of excitatory versus inhibitory neurons, axonal conduction delays and the number of synaptic connections affect a SNN model by observing the change in mean firing rate and polychronization. Our main focus is to examine the effect on the emergence of spatiotemporal time-locked patterns, known as polychronous groups (PNGs). We show that the number of PNGs varies dramatically with a changing proportion of inhibitory neurons, that they increase exponentially as the number of synaptic connections is increased and that they decrease as the maximum axonal delays in the network increases. Our findings show that if we are to use SNNs and PNGs to model cognitive functions we must take into account these critical parameters.
\end{abstract}

Keywords: Spiking neural networks, polychronization

\section{Introduction}

Neurons generate electric action potentials (spikes) that are used to transmit information to other cells via connections called synapses [1]. Axonal conduction delays - the time required for a spike to travel through neural axons - seems to lead to asychronous firing patterns called polychronous groups (PNGs) [2] The spatiotemporal behaviour of these patterns allow neurons to participate in multiple PNGs, resulting in numbers of PNGs far exceeding the number of neurons in a network. Current theories and models exploring PNGs have focused on typical neocortical parameters [3]. Exceptions include [4-7], which explored the impact of different topology networks, their connectivity, and different plasticity rules on the PNGs. However, if we are to fully understand PNGs, we must also observe the effect of varying network parameters on their incidence, especially since different brain regions have varying analogous parameters.

Brain networks of interconnecting systems exhibit a number of different physiological and biological parameters including the number of neurons, types of 
neurons and number of synapses. If we take working memory as an example, neuroanatomical investigations have associated areas such as the sensory and association cortex, prefrontal cortex, parietal lobes, thalamus and hippocampus [8]. The anatomical diversity of these areas can greatly affect experimental results and hence if as proposed [2] PNGs are a mechanism for information storage and transmission in the brain, we need to understand its effect.

In this paper, we will explore the current theory of polychronization with respect to parameters including synaptic lengths and delays, number of synapses and types of neuronal connectivity. We believe that this research may lead to uncovering the computational plausibility of theories that link PNGs to cognitive functions [3], and give us an insight in the information-processing mechanisms required for various memory tasks, as they are linked to distinct systems. We show for example that the physiological and biological differences of the hippocampus as opposed to other cortical areas can play a major role in the development and formation of PNGs.

\section{Method}

The main focus of this study is to examine how the number of polychronous groups varies when we change network parameters. For example, what effect does excitation have in the formation of such groups? How do conduction delays and synaptic connections change the dynamics of the model? We need to benchmark the model in order to explore future modelling capabilities. Current simulations exploring polychronization theory typically use a fixed set of parameters which may be unrealistic for a large number of brain areas. We want to test different parameters and compare general performance with observed 'equivalent' biological behaviour.

To explore these parameters we use the SNN model proposed in [9]. This can reproduce neuronal firing patterns of most known types of cortical neurons including regular spiking (RS), intrinsically bursting (IB), fast spiking (FB) among others, and yet is computationally simple and was used in Izhikevich [2] 's original model to find polychronous groups:

$$
\begin{aligned}
& v^{\prime}=0.04 v^{2}+5 v+140-u+I \\
& u^{\prime}=a(b v-u)
\end{aligned}
$$

with reset conditions,

$$
\text { if } v \geq+30 \mathrm{mV} \text {, then }\left\{\begin{array}{l}
v \leftarrow c \\
u \leftarrow u+d
\end{array}\right.
$$

Variable $v$ represents the membrane potential of the neuron; $u$ represents the membrane recovery variable, which accounts for the activation of $\mathrm{K}+$ ionic currents and inactivation of $\mathrm{Na}+$ ionic currents, and provides negative feedback to $v$; and $I$ represents synaptic or injected currents. The parameter $a$ describes the 
time scale of the recovery variable $u$. Smaller values result in slower recovery. The parameter $b$ describes the sensitivity of the recovery variable $u$ to the subthreshold fluctuations of the membrane potential $v$. Greater values couple $v$ and $u$ more strongly resulting in possible subthreshold oscillations and low-threshold spiking dynamics. The parameter $c$ describes the after-spike reset value of the membrane potential $v$ caused by the fast high-threshold $\mathrm{K}+$ conductances. The parameter $d$ describes after-spike reset of the recovery variable $u$ caused by slow high-threshold $\mathrm{Na}+$ and $\mathrm{K}+$ conductances.

Using this neuronal model, Izhikevich presented a minimal spiking network that exhibited time-locked asynchronous firing patterns with millisecond precision [2]. The network consisted of 1000 spiking neurons, 800 excitatory and 200 inhibitory with up to $20 \mathrm{~ms}$ axonal conduction delays for excitatory connections (1ms for inhibitory), each excitatory neuron connected to 100 random neurons (each inhibitory neuron connected to 100 excitatory only) and was subject to spike-timing dependent plasticity (STDP) [10,11]. In such a network, and the main concept behind polychronization as a theory, two neurons may fire at distinct times, yet their spikes arrive at a common postsynaptic neuron simultaneously due to the difference in conduction delays. The arrival of the spikes will cause the postsynaptic neuron to fire. The output spikes from the postsynaptic neurons may again arrive simultaneously at other neurons, causing further spike activations. This specific time-locked firing pattern of a chain of neurons is called a polychronous group (PNG).

\section{Experiments and Evaluation}

For our experiments we used a $\mathrm{C}++$ implementation of the spiking network with axonal conduction delays and STDP which is publicly available [2]. In all of our experiments we simulated 18,000 seconds of network activity. We repeated the simulations 10 times in order to obtain a mean for the number of groups and firing rate. The program simulates the dynamics of a spiking network with delays and STDP, then uses the algorithm for counting groups defined by Izhikevich [2], we consider various triplets of neurons firing with various patterns revealing all polychronous groups that arise from the triplets. In our experiments we consider multiple values for the ratio of excitatory to inhibitory (excitatory neurons up to 900 out of 100), up to 150 connections per neuron, conduction delays up to a maximum of $40 \mathrm{~ms}$, and an experiment on the minimum path required for a $\mathrm{PNG}$, in order to see the effect they have on the number of groups.

\subsection{Excitation versus Inhibition}

The functional architecture of neuronal networks is defined by the neurons and their connectivity. Cortical networks consist of two basic neural elements: excitatory principal cells and inhibitory interneurons. Research has suggested that in the cerebral cortex, excitatory synapses represent the majority of the synapses $(80 \%)$ [1]. In addition, there is evidence that areas such as the CA1 region of the 
hippocampus may have as many as 30,000 excitatory inputs and 1700 inhibitory inputs [12]. However, at this point we want to stress that a detailed distribution of excitatory as well as inhibitory inputs on the somata and dendrites of neural cells, not only in working memory related brain areas, but also in the whole neural system remains elusive [1]. Thus, our line of focus is not to create a direct mapping of the excitatory versus inhibitory population ratios of specific brain areas, but to get a general view of how excitatory and inhibitory inputs affect the generation of polychronous groups. In this experiment we change the ratio of excitatory to inhibitory neurons to see how it affects the number of groups, keeping the number of neurons and other parameters fixed.
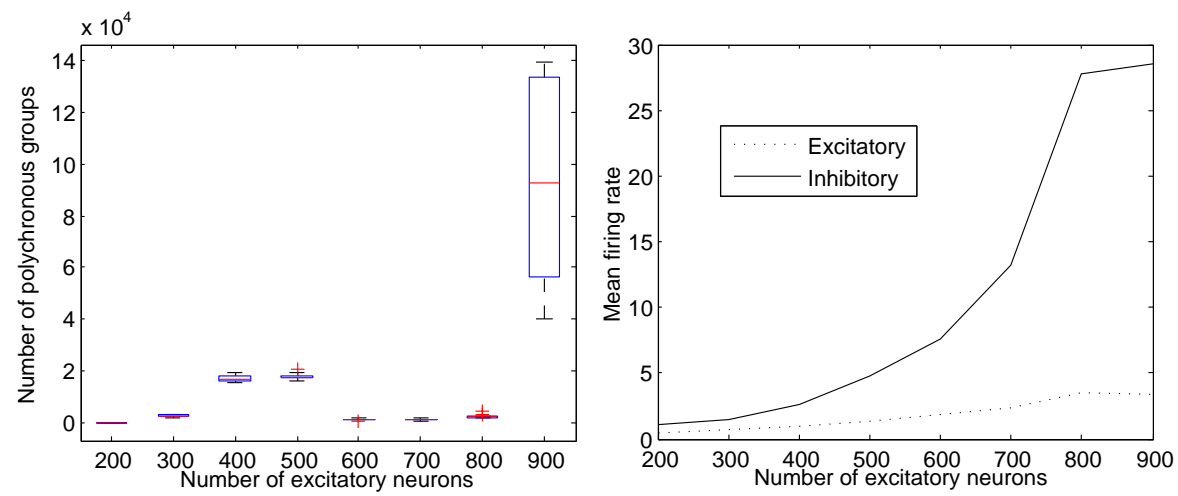

Fig. 1. Polychronous groups and firing rates versus the number of excitatory neurons. Boxplots in all figures denote the median, the $25^{\text {th }}$ and $75^{\text {th }}$ percentiles, extreme points and outliers $(+)$.

Fig. 1 shows a sudden growth in the number of PNGs for over 800 excitatory neurons which indicates that excitation increases the total number of polychronous groups. This suggests that there might be brain areas where polychronous groups emerge at quite different scales. If we attempt to map our results broadly to brain areas with different ratios of excitatory to inhibitory, then the prefrontal cortex (800:200), for example, is thought to produce far less polychronous groups than hippocampal areas (950:50). This perhaps fits with our understanding of the functionality each brain area has i.e. short-term memory sustainability in the prefrontal cortex and memory consolidation in the hippocampus [1].

In Fig. 1 we also note that there is a rise in the number of groups for 400 and 500 excitatory neurons followed by a fall for 600 to 800 . The depression in the number of groups coincides with the rapid rise of inhibitory firing rates, which levels off from 800 onwards. This suggests that the increased activity in the inhibitory neurons, which resonates at a ratio of 800 to 900 excitatory neurons, somehow suppresses activity sufficiently to prevent a larger number of groups forming.

\section{$3.2 \quad$ Number of Synaptic Connections}

A synapse, in addition to its ability to mediate different specific functions like STDP, has a relatively small size. The area of contact has a diameter of 0.5- 
$20 \mu \mathrm{m}$, and the presynaptic terminal's diameter is slightly larger [1]. Therefore, a large number of synapses can be packed into a limited space in brain areas. For example, in the cat visual cortex, $1 \mathrm{~mm}^{3}$ of grey matter contains approximately 50,000 neurons, with each neuron having an average of 6000 synapses, giving a total of $300 \times 10^{6}$ synapses [1]. Thus, if cortical areas consist of billions of neurons, then the synapses are in the order of trillions. In the polychronization model however, a value of 100 synapses per neuron is used. Consequently, in our experiments, we varied the number of synapses to explore how the number of polychronous groups is affected as we increase the number of synapses keeping the ratio excitatory to inhibitory neurons at 800:200 and the number of neurons and all other parameters fixed.
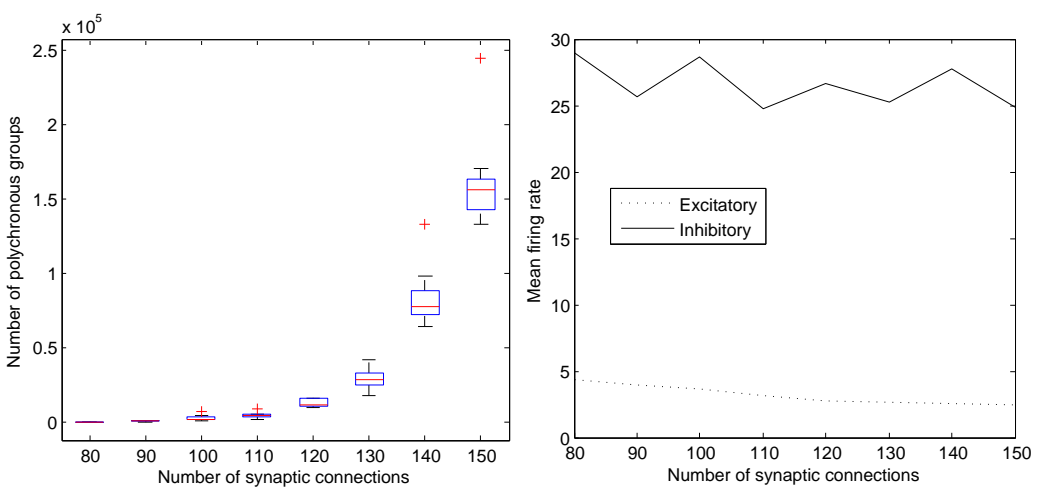

Fig. 2. Polychronous groups and firing rates versus the number of synaptic connections.

Fig. 2 shows that for over 120 connections the number of groups increases exponentially while the spike rates essentially remain constant. For less than 80 connections per neuron a few groups emerge. Most importantly, if we take into account the scale of increase compared to our previous experiment we can see that synaptic connections play a more important role in the formation of polychronous groups. We can see that if we increase the number of connections from 100 to 140 per neuron, the increase is in the order of 100,000 more polychronous groups that emerge. In addition, if we take into consideration that the number of connections quite probably far exceeds the number of neurons in a network, we can see how this number might scale up. On the other hand, in our previous experiment where we varied the number of excitatory versus inhibitory connections in the network, in order to reach an increase of the order of 100,000 of the number of polychronous groups we stretched the ratio to the maximum possible. This shows how important the number of synaptic connections is to the actual number of polychronous groups.

\subsection{Range of Axonal Delays}

Axonal conduction delays refer to the time required for an action potential to travel from its initiation site near the neuronal soma to the axon terminals, where synapses connect the soma with other neurons [1]. Evidence suggests 
that conduction time in the mammalian brain can reach from a few ms up to over 50ms [13]. In the polychronization model however, conduction delays had an upper limit of $20 \mathrm{~ms}$. In our experiment, we set the upper limit of axonal conduction delays to $40 \mathrm{~ms}$, to see again how the number of polychronous groups will decrease.
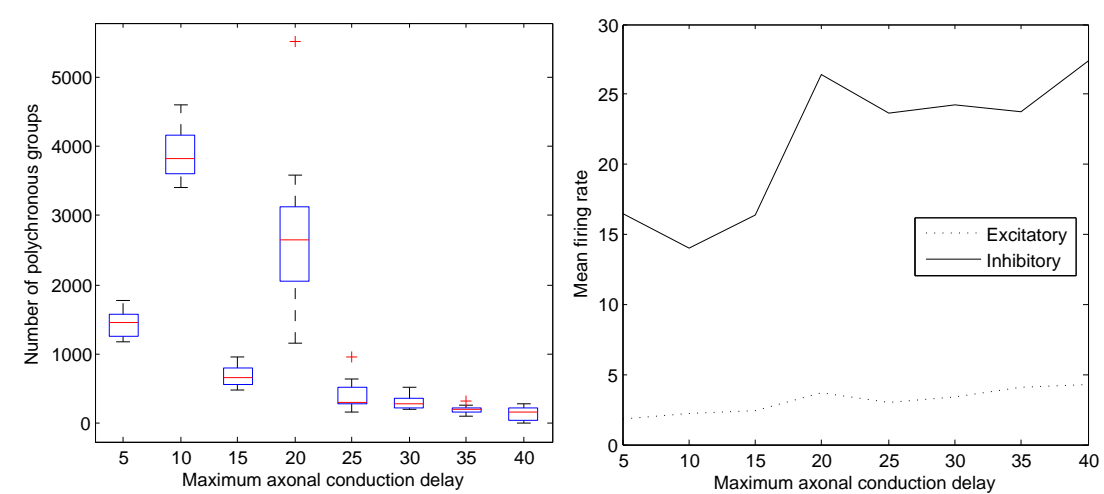

Fig. 3. Polychronous groups and firing rates versus the number of synaptic connections.

Fig. 3 shows that the number of polychronous groups decreases rapidly as the maximum axonal conduction delay increases. It appears that axonal conduction delays significantly affect the total number of polychronous groups. Most importantly however, even when we stretched the conduction delays to match the maximum experimental measurements, polychronous groups still emerged, even though they decreased while firing rates remained almost constant. There is some fluctuation for short delays ( 5 to $20 \mathrm{~ms}$ ), and this perhaps coincides with the lower firing rates of inhibitory neurons observed.

\subsection{Minimum Group Path}

This experiment, as opposed to the previous ones, does not involve variation of an anatomical parameter, but focuses on the minimum group path (the minimum length of the chain of neurons activated in a group) that actually defines such a polychronous group [2]. This is quite important because of the role of a polychronous group can have in cognitive computations. For example, polychronous groups may represent possible memory cues, in a way that every time the input is presented to the network, a polychronous group whose spike-timing pattern resembles the activated input [3]. Thus, the path of a group can actually be linked to the size or duration of the input stimulus or the memory cue.

Fig. 4 shows that the groups are decreasing rapidly as the minimum group path increases. If we take into consideration polychronous group path lengths above $25 \mathrm{~ms}$, no polychronous groups emerge at all. This is perhaps a limitation of the network size and further work is needed to determine if a larger network can sustain groups with larger paths. 


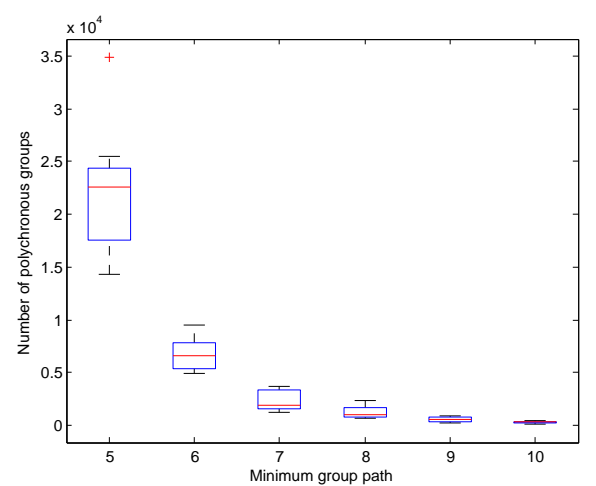

Fig. 4. Minimum group path length

\subsection{Conclusion}

Polychronization can be described in a few words as a systematic temporal firing activity and it is a newly proposed mechanism of neuronal encoding. Attempts have been made to assess its performance by investigating the effect of network topology on the recall of a PNG [4]. It is used in reservoir computing classification models [14]. It is also proposed as a working memory theory, where PNGs resemble memory cues which can be simultaneously sustained [3]. A simple neural network model with a minimal number of features was used for a similar study of polychronous groups, lacking however essential neural characteristics like STDP and inhibition, nonetheless producing similar results [5]. On the other hand, the effect of network topology on neuronal encoding based on polychronization was explored in [4], in which the results produced suggest that it is a crucial parameter, i.e. small-world networks favour information processing through polychronization. Also the precise type of STDP rule used may influence the number of PNGs [7].

In this paper we presented our current experimental results and drew conclusions regarding the appearance of polychronous groups. We varied parameters including the ratio of excitatory vs. inhibitory neurons, axonal conduction delays, the number of synaptic connections as well as the minimum group path of a PNG. These results show that the number of PNGs varies dramatically with a changing proportion of inhibitory neurons, that they increase exponentially as the number of synaptic connections is increased and that they decrease as the maximum axonal delays in the network increases.

Can we draw conclusions from polychronization experimental results about the human brain? It seems that certain parameters that vary from region to region are critical to the emergence of these groups. If as hypothesized in [2] that polychronous groups may represent memories and experience, could the group path length limit account for limitations observed such as working memory capacity [15], or how could this affect the sustainability of the polychronous groups in working memory tasks?

Our findings suggest that the emergence of polychronous groups can vary across analogous brain regions. Here, different ratios of excitatory to inhibitory 
neurons in the prefrontal cortex and the hippocampus suggest that a higher number of polychronous groups emerge in the hippocampus. It appears that the number of synaptic connections is the most important factor affecting the total number of polychronous groups.

Having established that parameter choice is critical to the emergence of PNGs, we will next turn our attention to whether PNGs can encode information which is sustainable. Here we are interested in whether SNNs can achieve stabilization of a specific temporal spiking activity such as in the spike-timing theory of working memory [3] which can then be used for working memory.

\section{References}

1. Shepherd, G.M.: The Organization ofthe Brain. Oxford University Press (2004)

2. Izhikevich, E.M.: Polychronization: Computation with spikes. Neural Computation 18(2) (2006) 245-282

3. Szatmáry, B., Izhikevich, E.M.: Spike-timing theory of working memory. PLoS Comput Biol 6(8) (2010) e1000879

4. Vertes, P.E., Duke, T.: Effect of network topology on neuronal encoding based on spatiotemporal patterns of spikes. HFSP Journal 4(3-4) (2010) 153-163

5. Maier, W.L., Miller, B.N.: A minimal model for the study of polychronous groups. Time 2 (2008) 8

6. Notley, S., Grüning, A.: Improved spike-timed mappings using a tri-phasic spike timing-dependent plasticity rule. In: Proceedings of the International Joint Conference on Neural Networks 2012. (Accepted)

7. Chrol-Cannon, J., Grüning, A., Jin, Y.: The emergence of polychronous groups under varying input patterns, plasticity rules and network connectivities. In: Proceedings of the International Joint Conference on Neural Networks 2012. (Accepted)

8. Cowan, N. In: An Embedded-Processes Model of Working Memory. Cambridge University Press (1999)

9. Izhikevich, E.M.: Simple model of spiking neurons. Neural Networks, IEEE Transactions on 14(6) (2003) 1569-1572

10. Bi, G.Q., Poo, M.M.: Synaptic modifications in cultured hippocampal neurons: Dependence on spike timing, synaptic strength, and postsynaptic cell type. The Journal of Neuroscience 18(24) (1998) 10464-10472

11. Song, S., Miller, K.D., Abbott, L.F.: Competitive hebbian learning through spike-timing-dependent synaptic plasticity. Nat Neurosci 3(9) (2000) 919-926 $10.1038 / 78829$.

12. Megías, M., Emri, Z., Freund, T.F., Gulyás, A.I.: Total number and distribution of inhibitory and excitatory synapses on hippocampal ca1 pyramidal cells. Neuroscience 102(3) (2001) 527-540

13. Swadlow, H.A.: Physiological properties of individual cerebral axons studied in vivo for as long as one year. Journal of Neurophysiology 54(5) (1985) 1346-1362

14. Paugam-Moisy, H., Martinez, R., Bengio, S.: Delay learning and polychronization for reservoir computing. Neurocomput. 71(7-9) (March 2008) 1143-1158

15. Miller, G.A.: The magical number seven, plus or minus two: Some limits on our capacity for processing information. Psychological Review 101(2) (1994) 343-352 